\title{
A SUBSTITUTE OF L'HOSPITAL'S RULE FOR MATRICES
}

\author{
W. KRATZ
}

\begin{abstract}
In this paper the following limit theorem is obtained: If $A$ and $B$ are $(n, n)$-matrices with $\operatorname{rank}\left(A^{T}, B^{T}\right)=n, A^{T} B=B^{T} A$, then $A(A+S B)^{-1} S \rightarrow 0$ as $S \rightarrow 0+$, i.e. $S \rightarrow 0$ where $S$ is symmetric and positive definite. Some applications of this result are given to linear algebra (the behavior of $(A+\lambda B)^{-1}$ as $\lambda \rightarrow 0$ ) and to differential equations (the asymptotic behavior of Hamiltonian systems and of selfadjoint differential equations of even order).
\end{abstract}

1. Introduction. Let $A(x), B(x), C(x)$ be real $(n, n)$-matrices such that $A(x)$, $B(x), C(x)$ are continuous, $B(x), C(x)$ are symmetric, and $B(x)$ is nonnegative definite for $x \in \mathbf{R}$. We consider $(n, n)$-matrices $U(x), V(x)$ which solve the Hamiltonian system $U^{\prime}=A U+B V, V^{\prime}=C U-A^{T} V$ with $U^{T}(x) V(x) \equiv V^{T}(x) U(x)$ and $\operatorname{rank}\left(U^{T}(x), V^{T}(x)\right) \equiv n$ (so-called conjoined basis [1, Definition 1.2]). Moreover, we assume that the Hamiltonian system is normal $[\mathbf{1}$, Lemma $5 ; 2]$, i.e. the focal points of $U(x)$ (those $x$ where $U(x)$ is singular) are isolated. Observe that if $U^{-1}(x)$ exists, then $Q(x):=V(x) U^{-1}(x)$ is a symmetric matrix-function solving the Riccati equation $Q^{\prime}+A^{T} Q+Q A+Q B Q-C=0[\mathbf{1}, \mathbf{2}, \mathbf{3}]$. These differential systems are the basis for variational analysis of the second variation or for Sturm-Liouville eigenvalue problems. The following identity (due to Picone) is an essential tool, e.g. for deriving comparison and oscillation results:

$$
(d / d x) \eta^{T} Q \eta=\eta^{T} C \eta+\varsigma^{T} B \varsigma-(\varsigma-Q \eta)^{T} B(\varsigma-Q \eta)
$$

whenever $U^{-1}(x)$ exists and whenever $\eta(x), \varsigma(x)$ belong to $C_{1}(\mathbf{R})$ with $\eta^{\prime}=A \eta+B \varsigma$ $[\mathbf{1}$, Theorem 7 with $\alpha=0 ; \mathbf{2}, \mathbf{5}]$. If $U(x)$ is singular for some $x_{0} \in \mathbf{R}$, then we need to know the behavior of $Q(x)$ as $x \rightarrow x_{0}$ when applying this identity. It is shown in [1] that

$$
\lim _{x \rightarrow x_{0}} u^{T}(x) Q(x) u(x)=d^{T} U^{T}\left(x_{0}\right) V\left(x_{0}\right) d
$$

for any vector-solution $u(x), v(x)$ of the Hamiltonian system with $u\left(x_{0}\right)=U\left(x_{0}\right) d$ for some $d \in \mathbf{R}^{n}$. In the scalar case, i.e. $n=1$, this limit theorem follows from l'Hospital's rule, and in this sense the theorem and also our result below may be considered as a substitute of l'Hospital's rule for matrices.

In this paper we derive a limit theorem for matrices where no differential equation is involved and which generalizes the result above. More precisely, we prove that $A(A+S B)^{-1} S \rightarrow 0$ as $S \rightarrow 0+$, i.e., $S \rightarrow 0$ and $S$ is symmetric and positive definite, whenever $A$ and $B$ are $(n, n)$-matrices with $\operatorname{rank}\left(A^{T}, B^{T}\right)=n, A^{T} B=B^{T} A$

Received by the editors January 22, 1986.

1980 Mathematics Subject Classification (1985 Revision). Primary 15A24, 15A45; Secondary 34B25, 34C11, 34E99.

Key words and phrases. Hamiltonian system, Riccati equation, symmetric matrices, matrix functions, Picone identity, focal points. 
(Theorem 1 of $\S 2$ ). In $\S 3$ and $\S 4$ we give some applications of this theorem to linear algebra (we study the behavior of $(A+\lambda B)^{-1}$ as $\lambda \rightarrow 0$ ) and to differential equations (we derive the limit result above and also the asymptotic behavior of solutions of $L(y)=\lambda r y$ as $\lambda \rightarrow-\infty$, where $L(y)$ is a scalar, selfadjoint differential operator of even order). In $\S 5$ we discuss the assumptions on $A, B$, and $S$ made in Theorem 1.

2. Main results. We use the following notation. If $A$ and $B$ are $(n, n)$-matrices, then $A \leq B$ (resp., $A<B$ ) means that $A$ and $B$ are symmetric and $B-A$ is nonnegative definite (resp., positive definite). We denote by $A^{T}$ the transpose of $A$, by $I$ the identity matrix, and by $\|\cdot\|$ the Euclidean norm of a vector or the induced matrix-norm (i.e., $\max _{d \neq 0}\|A d\| /\|d\|$ ) of a matrix. Moreover, we use a result which is included in $[\mathbf{1}$, Proposition A 1 (ii), (iii), Proposition 1, and formula $\left.\left(7^{\prime}\right)\right]$.

Proposition 1. Suppose that $A$ and $B$ are $(n, n)$-matrices such that

$$
\operatorname{rank}\left(A^{T}, B^{T}\right)=n, \quad A^{T} B=B^{T} A .
$$

Then the following assertions hold:

(i) $\operatorname{kernel}\left(A^{T} B\right)=\operatorname{kernel}(A) \oplus \operatorname{kernel}(B)$, where $\oplus$ denotes a direct sum;

(ii) $A+S B$ is regular for all $S$ with $0<S \leq \varepsilon I$, if $\varepsilon>0$ is sufficiently small;

(iii) there exist matrices $A_{1}, B_{1}$ such that the following identities hold:

$$
\begin{aligned}
& A^{T} B=B^{T} A, \quad A_{1}^{T} B_{1}=B_{1}^{T} A_{1}, \quad A A_{1}^{T}=A_{1} A^{T}, \quad B B_{1}^{T}=B_{1} B^{T}, \\
& A B_{1}^{T}-A_{1} B^{T}=A^{T} B_{1}-B^{T} A_{1}=I .
\end{aligned}
$$

Moreover, $A_{1}$ and $B_{1}$ may be chosen to be regular.

REMARK. Matrices $A_{1}, B_{1}$ according to (iii) are constructed in the proof of $[\mathbf{1}$, Proposition 1] by $A_{1}=-(B+\varepsilon A) K^{-1}, B_{1}=(A-\varepsilon B) K^{-1}$ with $K=A^{T} A+B^{T} B$ and with sufficiently small $\varepsilon>0$.

The main result of this paper is given by

THEOREM 1. If $A$ and $B$ are $(n, n)$-matrices with (1), then

$$
\lim _{S \rightarrow 0+} A(A+S B)^{-1} S=0,
$$

where $S \rightarrow 0+$ stands for $S \rightarrow 0, S$ is a symmetric and positive definite $(n, n)$ matrix.

This theorem is a direct consequence of

LEMMA 1. Given $(n, n)$-matrices $A, B$ with (1), there exist $\varepsilon>0$ and $c>0$ such that

$$
0 \leq A(A+S B)^{-1} S \leq S+c S^{2} \text { for all } S \text { with } 0<S \leq \varepsilon I .
$$

ProOF. We assume throughout that $\varepsilon>0$ is sufficiently small. Hence, $Q(S):=$ $A(A+S B)^{-1} S$ exists for $0<S \leq \varepsilon I$ by Proposition 1(ii). Since

$$
\left(A^{T}+B^{T} S\right) S^{-1} Q(S) S^{-1}(A+S B)=A^{T} S^{-1} A+B^{T} A
$$

(use $S^{T}=S$ ) the matrix $Q(S)$ is symmetric and $\geq 0$ for all $0<S \leq \varepsilon I$ (use that $c^{T} B^{T} A c=c_{1}^{T} B^{T} A c_{1}$, if $c=c_{1}+c_{2}$ with $c_{1} \in \operatorname{image}\left(A^{T}\right), c_{2} \in \operatorname{kernel}(A)$ which is 
orthogonal to $c_{1}$, and use $B^{T} A=A^{T} B$ ). Now fix $\varepsilon>0$ and $S$ with $0<S \leq \varepsilon I$, and let $S(x):=S+x(\varepsilon I-S)$. Then $S=S(0) \leq S(x) \leq S(1)=\varepsilon I$ for $0 \leq x \leq 1$. Since $Q(S)=S+S\left\{-B(A+S B)^{-1}\right\} S$ we obtain that $-B(A+S(x) B)^{-1}$ is symmetric. Moreover, if $0 \leq x \leq 1$, then

$$
\frac{d}{d x}\left\{-B(A+S(x) B)^{-1}\right\}=\left\{B(A+S(x) B)^{-1}\right\}(\varepsilon I-S)\left\{B(A+S(x) B)^{-1}\right\} \geq 0 .
$$

This implies that

$$
0 \leq Q(S) \leq S+S\left\{-B(A+S(1) B)^{-1}\right\} S \leq S+c S^{2},
$$

where $c>0$ is chosen such that $-B(A+\varepsilon B)^{-1} \leq c I$.

A first consequence of Theorem 1 is

COROLLARY 1. Under the assumptions of Theorem 1 let $S_{0}$ be any symmetric $(n, n)$-matrix. Then

$$
\lim _{S \rightarrow S_{0}+}\left(A+S_{0} B\right)(A+S B)^{-1}\left(A+S_{0} B\right)=A+S_{0} B,
$$

where $S \rightarrow S_{0}+$ means $S \rightarrow S_{0}$ with $S>S_{0}$.

Proof. We have $A+S B=A+S_{0} B+\left(S-S_{0}\right) B, \operatorname{rank}\left(A^{T}+B^{T} S_{0}, B^{T}\right)=$ $\operatorname{rank}\left(A^{T}, B^{T}\right)=n$, and $\left(A^{T}+B^{T} S_{0}\right) B=A^{T} B+B^{T} S_{0} B$ is symmetric by (1). Hence

$$
\begin{aligned}
& \left(A+S_{0} B\right)(A+S B)^{-1}\left(A+S_{0} B\right) \\
& \quad=A+S_{0} B-\left(A+S_{0} B\right)\left(A+S_{0} B+\left(S-S_{0}\right) B\right)^{-1}\left(S-S_{0}\right) B \rightarrow A+S_{0} B
\end{aligned}
$$

as $S \rightarrow S_{0}+$ by Theorem 1 with $A+S_{0} B$ instead of $A$.

In applications to differential equations ([1, Theorem 4] or Propositions 4, 5 below) Theorem 1 is used in the following form.

COROLLARY 2. Suppose that the assumptions of Theorem 1 hold and that $A_{1}, B_{1}$ are chosen such that (2) holds (Proposition 1(iii)). Then

$$
\lim _{S \rightarrow 0+} A(A+S B)^{-1}\left(A_{1}+S B_{1}\right) A^{T}=A_{1} A^{T} .
$$

Proof. We have $\lim _{S \rightarrow 0+} A(A+S B)^{-1} A=A$ by Corollary 1 . Hence

$$
\lim _{S \rightarrow 0+} A(A+S B)^{-1}\left(A_{1}+S B_{1}\right) A^{T}=\lim _{S \rightarrow 0+} A(A+S B)^{-1} A A_{1}^{T}=A_{1} A^{T}
$$

since $A_{1} A^{T}=A A_{1}^{T}$ by (2).

This corollary shows that for $c=A^{T} d \in \operatorname{image}\left(A^{T}\right)$,

$$
\lim _{S \rightarrow 0+} c^{T}(A+S B)^{-1}\left(A_{1}+S B_{1}\right) c
$$

exists and equals $d^{T} A_{1} A^{T} d$. Actually, these $c$ 's are the only ones for which the limit exists. This fact is stated in the following theorem. We omit the proof since it follows in the same way as Corollary 7 of [1] (using essentially the minimummaximum principle, Proposition 1(i), and the fact that

$$
P(S):=(A+S B)^{-1}\left(A_{1}+S B_{1}\right)
$$

is strictly increasing, i.e., $P\left(S_{1}\right)<P\left(S_{2}\right)$ for $\left.0<S_{1}<S_{2} \leq \varepsilon I\right)$. 
THEOREM 2. Suppose that the assumptions of Theorem 1 hold and that $A_{1}, B_{1}$ are chosen such that (2) holds. Then

$$
\lim _{S \rightarrow 0+} c^{T}(A+S B)^{-1}\left(A_{1}+S B_{1}\right) c=-\infty \quad \text { for all } c \notin \operatorname{image}\left(A^{T}\right) .
$$

REMARK. The assertions of Theorems 1 and 2 with $-\infty$ instead of $+\infty$ hold also for $S \rightarrow 0-$ (replace $B$ by $-B$ and $A_{1}$ by $-A_{1}$ ). But the limits have to be one-sided as is shown in $\S 5$.

3. Applications to linear algebra. The result in this section is

Proposition 2. Let $A$ and $B$ be $(n, n)$-matrices with (1). Then

$$
Q=\lim _{\lambda \rightarrow 0} \lambda(A+\lambda B)^{-1}
$$

exist, and the matrices $Q$ and $P:=B Q=\lim _{\lambda \rightarrow 0} \lambda B(A+\lambda B)^{-1}$ are characterized by the properties

(i) $P$ is symmetric, idempotent, $\operatorname{rank}(P)=n-\operatorname{rank}(A), P A=0$;

(ii) $Q=\left(A^{T} A+B^{T} B\right)^{-1} B^{T} P$.

Proof. By Lemma 1 (with $S=\lambda I$ ) there exist $\varepsilon>0, c>0$ such that $0 \leq$ $A(A+\lambda B)^{-1} \leq I+\lambda c I$, and $0 \leq A(A-\lambda B)^{-1} \leq I+\lambda c I$ (with $-B$ instead of $B$ ) for all $0<\lambda \leq \varepsilon$. Hence, the matrix-elements of $A(A+\lambda B)^{-1}$ are bounded for $0<|\lambda| \leq \varepsilon$. Since these matrix-elements are rational functions of $\lambda$,

$$
\lim _{\lambda \rightarrow 0} A(A+\lambda B)^{-1}=I-P
$$

exists, and then $\lim _{\lambda \rightarrow 0} \lambda B(A+\lambda B)^{-1}=P$ with a symmetric matrix $P$. Since $K:=A^{T} A+B^{T} B$ is regular by (1), it follows that

$$
\lambda(A+\lambda B)^{-1}=K^{-1} \lambda\left\{A^{T} A(A+\lambda B)^{-1}+B^{T} B(A+\lambda B)^{-1}\right\} \rightarrow K^{-1} B^{T} P=: Q .
$$

Then, of course, $P=B Q$, and (ii) is proved. Next, we have

$$
Q A=\lim _{\lambda \rightarrow 0} \lambda(A+\lambda B)^{-1} A=\lim _{\lambda \rightarrow 0}\left(\lambda I-\lambda^{2}(A+\lambda B)^{-1} B\right)=0 .
$$

Hence $P A=0$, and since $P$ is symmetric, we obtain that $P\left(A A^{T}\right)=0=\left(A A^{T}\right) P$. Therefore $P$ and $A A^{T}$ are simultaneously orthogonally diagonalizable $[4$, Theorem 6.2], i.e. there exists an orthogonal matrix $U$ such that

$$
U^{T}\left(A A^{T}\right) U=\left(\begin{array}{cc}
D_{1} & 0 \\
0 & 0
\end{array}\right), \quad D_{1}=\operatorname{diag}\left(\lambda_{1}, \ldots, \lambda_{k}\right), \quad \lambda_{i}>0, k=\operatorname{rank}(A),
$$

and

$$
U^{T} P U=\left(\begin{array}{cc}
0 & 0 \\
0 & D_{2}
\end{array}\right), \quad D_{2}=\operatorname{diag}\left(\mu_{k+1}, \ldots, \mu_{n}\right) .
$$

Finally, if $A A^{T} c=0$, then

$$
\begin{aligned}
0 & =\left(A^{T}+\lambda B^{T}\right)^{-1} A^{T} c=A(A+\lambda B)^{-1} c \quad \text { (by symmetry) } \\
& =\lim _{\lambda \rightarrow 0} A(A+\lambda B)^{-1} c=c-P c .
\end{aligned}
$$

Hence, every $c \in \operatorname{kernel}\left(A A^{T}\right), c \neq 0$, is an eigenvector of $P$ with eigenvalue 1 . This implies that $\mu_{k+1}=\cdots=\mu_{n}=1$, which completes the proof. 
REMARKS. Observe first that $P$ is uniquely determined by property (i), more precisely: $P=U^{T} J U$, where $U$ is an orthogonal matrix with (3) and where $J=$ $\left(\begin{array}{ll}0 & 0 \\ 0 & I\end{array}\right)$ with $(n-k, n-k)$-identity matrix $I$. Thus, the matrix $P$ depends on $A$ only (not on $B$ ). Observe moreover, that for every $(n, n)$-matrix $A$ there exists a matrix $B$ such that (1) holds. Finally, we note as a consequence of Proposition 2 that $Q A=A Q=0$ and $\operatorname{rank}(Q)=\operatorname{rank}(P)=n-\operatorname{rank}(A)$.

4. Applications to differential equations. First, we study the local behavior of matrix solutions of Hamiltonian systems resp. of Riccati equations as discussed in the introduction. We consider $(n, n)$-matrices $U(x), V(x)$ which solve the Hamiltonian system

$$
U^{\prime}=A U+B V, \quad V^{\prime}=C U-A^{T} V,
$$

where we assume that

$$
A(x), B(x), C(x) \text { are continuous, } B(x), C(x) \text { are symmetric, }
$$

$B(x)$ is nonnegative definite for $x \in \mathbf{R}$, and where the differential system is normal $[\mathbf{1}]$.

Moreover, we assume that $U(x), V(x)$ is a conjoined basis of (4) [1, Definition 1, 2], i.e.,

$$
\operatorname{rank}\left(U^{T}(x), V^{T}(x)\right) \equiv n, \quad U^{T}(x) V(x) \equiv V^{T}(x) U(x)
$$

holds.

Our first result is

Proposition 3. If $B\left(x_{0}\right)$ is regular (for some $x_{0} \in \mathbf{R}$ ), then

$$
\lim _{x \rightarrow x_{0}}\left(x-x_{0}\right) U^{-1}(x)=Q
$$

exists, and

$Q=\left(U^{T}\left(x_{0}\right) B^{-1}\left(x_{0}\right) U\left(x_{0}\right)+V^{T}\left(x_{0}\right) B\left(x_{0}\right) V\left(x_{0}\right)\right)^{-1} V^{T}\left(x_{0}\right) \sqrt{B\left(x_{0}\right)} P \sqrt{B^{-1}\left(x_{0}\right)}$,

where the matrix $P$ is characterized by

$$
\begin{aligned}
& P \text { is symmetric, idempotent, } \operatorname{rank}(P)=n-\operatorname{rank}\left(U\left(x_{0}\right)\right), \\
& \text { and } P \sqrt{B^{-1}\left(x_{0}\right)} U\left(x_{0}\right)=0 .
\end{aligned}
$$

In particular $Q U\left(x_{0}\right)=0$ and $\lim _{x \rightarrow x_{0}}\left(x-x_{0}\right) U^{-1}(x) U\left(x_{0}\right)=0$ hold.

Proof. By our assumptions $S:=B\left(x_{0}\right)>0$, and (4) implies

$$
\begin{aligned}
U(x) & =U\left(x_{0}\right)+\left(x-x_{0}\right) U^{\prime}\left(x_{0}\right)+o(1)\left(x-x_{0}\right) \\
& =(I+o(1))\left(U\left(x_{0}\right)+\left(x-x_{0}\right) S V\left(x_{0}\right)\right) \text { as } x \rightarrow x_{0} .
\end{aligned}
$$

Put $\tilde{A}:=\sqrt{S^{-1}} U\left(x_{0}\right), \tilde{B}=\sqrt{S} V\left(x_{0}\right)$. Then $\tilde{A}, \tilde{B}$ satisfy (1), and

$$
U^{-1}(x)=\left(\tilde{A}+\left(x-x_{0}\right) \tilde{B}\right)^{-1} \sqrt{S^{-1}}(I+o(1)) \text { for } x \rightarrow x_{0} .
$$

Now, our assertions follow from Proposition 2.

In case $B\left(x_{0}\right)$ is singular, we have the following result [1, Corollaries 6 and 7 , which we mentioned in the introduction. 
PROPOSITION 4. If $u_{i}(x), v_{i}(x)(i=1,2)$ are vector-solutions of $(4)$, then

$$
\lim _{x \rightarrow x_{0}+} u_{1}^{T}(x) V(x) U^{-1}(x) u_{1}(x)=+\infty
$$

if $u_{1}\left(x_{0}\right) \notin \operatorname{image}\left(U\left(x_{0}\right)\right)$ and

$$
\lim _{x \rightarrow x_{0}} u_{1}^{T}(x) V(x) U^{-1}(x) u_{2}(x)=c_{1}^{T} V^{T}\left(x_{0}\right) U\left(x_{0}\right) c_{2}
$$

if $u_{i}\left(x_{0}\right)=U\left(x_{0}\right) c_{i} \in \operatorname{image}\left(U\left(x_{0}\right)\right)$ for $i=1,2$.

PROOF. Let $U_{i}, V_{i}(i=1,2)$ solve $(4)$ with the initial conditions $U_{1}\left(x_{0}\right)=$ $V_{2}\left(x_{0}\right)=0, V_{1}\left(x_{0}\right)=-U_{2}\left(x_{0}\right)=I[\mathbf{1}$, formula $(8)]$, and put $S(x)=-U_{2}^{-1}(x) U_{1}(x)$ for $|x| \leq \varepsilon$ ( $\varepsilon>0$ small enough). Then

$$
S^{\prime}(x)=U_{2}^{-1}(x) B(x)\left(U_{2}^{-1}(x)\right)^{T} \geq 0
$$

(note, $U_{1}^{T} V_{2}-V_{1}^{T} U_{2} \equiv I$ by the Wronskian identity [1, Lemma 2]), and therefore $S(x) \rightarrow 0$ as $x \rightarrow x_{0}+\left(S(x)>0\right.$ for $x>x_{0}$ by normality). Moreover, let $\tilde{U}, \tilde{V}$ solve (4), where the initial conditions are chosen according to Proposition 1(iii), such that $\tilde{U}^{T} \tilde{V} \equiv \tilde{V}^{T} \tilde{U}, U^{T} \tilde{V}-V^{T} \tilde{U} \equiv I$ holds. Then

$$
U(x)=U_{1}(x) V\left(x_{0}\right)-U_{2}(x) U\left(x_{0}\right), \quad \tilde{U}(x)=U_{1}(x) \tilde{V}\left(x_{0}\right)-U_{2}(x) \tilde{U}\left(x_{0}\right),
$$

and therefore

$$
U^{-1}(x) \tilde{U}(x)=\left(U\left(x_{0}\right)+S(x) V\left(x_{0}\right)\right)^{-1}\left(\tilde{U}\left(x_{0}\right)+S(x) \tilde{V}\left(x_{0}\right)\right) .
$$

Now, Corollary 2 and Theorem 2 imply that

$$
\lim _{x \rightarrow x_{0}} U\left(x_{0}\right) U^{-1}(x) \tilde{U}(x) U^{T}\left(x_{0}\right)=\tilde{U}\left(x_{0}\right) U^{T}\left(x_{0}\right),
$$

and

$$
\lim _{x \rightarrow x_{0}+} c^{T} U^{-1}(x) \tilde{U}(x) c=-\infty
$$

for all $c \notin$ image $\left(U^{T}\left(x_{0}\right)\right)$. This result is contained in Theorem 4 and Corollary 7 of [1] , and it implies our assertions as is shown essentially in [1, Corollary 6].

Finally we give an application to the asymptotic behavior (as $\lambda \rightarrow-\infty$ ) of solutions of the differential equation

$$
L(y)=\lambda r y,
$$

where $L(y)$ is a selfadjoint differential operator of even order, i.e.

$$
L(y)=\sum_{\nu=0}^{n}(-1)^{\nu}\left(r_{\nu} y^{(\nu)}\right)^{(\nu)}
$$

and where we assume that

$$
r_{\nu} \in C_{\nu}(\mathbf{R}), \quad \nu=0, \ldots, n, r \in C(\mathbf{R}), \text { and } r>0, r_{n}>0 \text { on } \mathbf{R}
$$

holds. For any function $y \in C_{2 n-1}(\mathbf{R})$ we define column-vectors $u(x)=\left(u_{k}(x)\right)$, $v(x)=\left(v_{k}(x)\right)$ by $[\mathbf{1} ; \mathbf{2}$, p. 309]

$$
u_{k}(x)=y^{(k)}(x), \quad v_{k}(x)=\sum_{\nu=k+1}^{n}(-1)^{\nu-k-1}\left(r_{\nu} y^{(\nu)}\right)^{(\nu-k-1)} \quad \text { for } k=0, \ldots, n-1 .
$$


Then $u, v$ solve a corresponding Hamiltonian system (4) with (5) iff $y$ solves (8) [1, 2]. Now, suppose that the functions $y_{i j}(x)(i=1, \ldots, n, j=1,2)$ with corresponding columns $u_{i j}(x), v_{i j}(x)$ are solutions of (8) with the initial conditions

$$
\begin{aligned}
& U_{j}\left(x_{0}\right)=\left(u_{1 j}\left(x_{0}\right), \ldots, u_{n j}\left(x_{0}\right)\right)=A_{j} \\
& V_{j}\left(x_{0}\right)=\left(v_{1 j}\left(x_{0}\right), \ldots, v_{n j}\left(x_{0}\right)\right)=B_{j} \\
& \quad \text { with } A_{j}^{T} B_{j}=B_{j}^{T} A_{j}, A_{1}^{T} B_{2}-B_{1}^{T} A_{2}=I \text { for } j=1,2
\end{aligned}
$$

(see Proposition 1(iii)). Then, of course, $U_{j}(x)=U_{j}(x ; \lambda)$, and the following holds.

Proposition 5. If $x>x_{0}$, then

$$
\lim _{\lambda \rightarrow-\infty} A_{1} U_{1}^{-1}(x ; \lambda) U_{2}(x ; \lambda) A_{1}^{T}=A_{2} A_{1}^{T}
$$

and

$$
\lim _{\lambda \rightarrow-\infty} c^{T} U_{1}^{-1}(x ; \lambda) U_{2}(x, \lambda) c=-\infty \quad \text { for all } c \notin \operatorname{image}\left(A_{1}^{T}\right) .
$$

PROOF. Fix $x>x_{0}$, and consider the solutions $\tilde{y}_{i j}(x)$ of (8) with the initial conditions $\tilde{U}_{1}\left(x_{0}\right)=\tilde{V}_{2}\left(x_{0}\right)=0, \tilde{V}_{1}\left(x_{0}\right)=-\tilde{U}_{2}\left(x_{0}\right)=I$ (compare the proof of Proposition 4). Then $\lim _{\lambda \rightarrow-\infty} \tilde{U}_{1}^{-1}(x ; \lambda) \tilde{U}_{2}(x ; \lambda)=-\infty$, i.e.

$$
S(\lambda):=-\tilde{U}_{2}(x ; \lambda) \tilde{U}_{1}^{-1}(x ; \lambda) \rightarrow 0+\text { as } \lambda \rightarrow-\infty,
$$

by $\left[\mathbf{1}\right.$, Theorem 11]. The initial conditions imply $U_{j}(x)=\tilde{U}_{1}(x) B_{j}-\tilde{U}_{2}(x) A_{j}$ for $j=1,2$, and therefore

$$
U_{1}^{-1}(x ; \lambda) U_{2}(x ; \lambda)=\left(A_{1}+S(\lambda) B_{1}\right)^{-1}\left(A_{2}+S(\lambda) B_{2}\right) .
$$

Now, our assertions follow from Corollary 2 and Theorem 2.

REMARK. Observe that $U_{j}, V_{j}(j=1,2)$ form a fundamental system of the Hamiltonian system (corresponding to (8)), and therefore every $u(x)=\left(y^{(k)}(x)\right)$, where $y(x)$ solves $(8)$, can be expressed in terms of $U_{1}, U_{2}$, i.e. $u(x)=U_{1}(x) c_{1}+$ $U_{2}(x) c_{2}$ for certain $c_{1}, c_{2} \in \mathbf{R}^{n}$.

5. Examples and concluding remarks. In this section we discuss the assumptions of Theorem 1 and show that they are all essential for the limit to exist.

(i) The assumptions (1) on the matrices $A$ and $B$ are essential, since $(A+S B)^{-1}$, of course, does not exist if $\operatorname{rank}\left(A^{T}, B^{T}\right)<n$. Moreover, if

$$
A=\left(\begin{array}{lll}
1 & 0 & 0 \\
0 & 1 & 0 \\
0 & 0 & 0
\end{array}\right), \quad B=\left(\begin{array}{lll}
0 & 0 & 0 \\
0 & 0 & 1 \\
0 & 0 & 0
\end{array}\right), \quad S(x)=\left(\begin{array}{ccc}
x^{3} / 3 & -x^{2} / 2 & 0 \\
-x^{2} / 2 & x & 0 \\
0 & 0 & x
\end{array}\right),
$$

then $\operatorname{rank}\left(A^{T}, B^{T}\right)=3, S(x)>0$ for $x>0$, but $A^{T} B \neq B^{T} A$. A simple calculation shows that $A+S(x) B$ is singular for all $x \in \mathbf{R}$.

(ii) The limit in Theorem 1 has to be one-sided in general. This follows from the example

$$
A=\left(\begin{array}{ll}
1 & 0 \\
1 & 0
\end{array}\right), \quad B=\left(\begin{array}{cc}
0 & 1 \\
0 & -1
\end{array}\right), \quad S(x)=\left(\begin{array}{cc}
x & 0 \\
0 & -x
\end{array}\right) .
$$

Then (1) holds, $S(x)$ is symmetric but indefinite for $x \neq 0$. Again, $A+S(x) B$ is singular for $x \in \mathbf{R}$. 
(iii) The matrix $S$ has to be symmetric, since if

$$
A=\left(\begin{array}{lll}
0 & 0 & 0 \\
0 & 0 & 1 \\
0 & 1 & 0
\end{array}\right), \quad B=I, \quad S(x)=\left(\begin{array}{ccc}
x^{3} & -x & 0 \\
x-x^{3} & x & 0 \\
0 & 0 & x
\end{array}\right),
$$

then (1) holds, $c^{T} S(x) c>0$ for all $c \in \mathbf{R}, c \neq 0$ and $0<x<2$, but again, $A+S(x) B$ is singular on $\mathbf{R}$.

Of course, in all examples, $S(x)$ may be changed 'slightly' so that $A+S(x) B$ is regular for $0<x \leq \varepsilon(\varepsilon>0$ small $)$, but the limit in Theorem 1 does not exist as $x \rightarrow 0+$.

Finally we give an example to show that no factor in $A(A+S B)^{-1} S$ can be dropped (as e.g. in the special case of $\S 3$ ). Put

$$
A=\left(\begin{array}{ll}
0 & 0 \\
1 & 0
\end{array}\right), \quad B=\left(\begin{array}{ll}
0 & 1 \\
1 & 0
\end{array}\right), \quad S(x)=\left(\begin{array}{cc}
x^{7} & x^{4} / 2 \\
x^{4} / 2 & x
\end{array}\right) .
$$

Then (1) holds and $S(x)>0$ for $x>0$. Hence, Theorem 1 applies, so that the matrices $A(A+S(x) B)^{-1} S(x), A(A+S(x) B)^{-1} A, S(x) B(A+S(x) B)^{-1} A$, $S(x) B(A+S(x) B)^{-1} S(x)$ tend to a limit as $x \rightarrow 0+$. But, in this example, the matrices $A(A+S(x) B)^{-1}, S(x)(A+S(x) B)^{-1} A$, and then also $S(x) B(A+S(x) B)^{-1}$, $S(x)(A+S(x) B)^{-1} S(x)$ do not converge as $x \rightarrow 0+$.

\section{REFERENCES}

1. W. Kratz and A. Peyerimhoff, A treatment of Sturm-Liouville eigenvalue problems via Picone's identity, Analysis 5 (1985), 97-152.

2. W. T. Reid, Ordinary differential equations, Wiley, New York, 1971.

3. __ Riccati differential equations, Academic Press, New York, 1972.

4. R. R. Stoll and E. T. Wong, Linear algebra, Academic Press, New York, 1969.

5. C. A. Swanson, Picone's identity, Rend. Mat. (6) 8 (1975), 373-397.

Universität Ulm, Abteilung Mathematik V, D-7900 Ulm, Oberer Eselsberg, FEDERAL REPUBLIC OF GERMANY 